\title{
Difficulties of an infrared extension of differential renormalization
}

\author{
L.V.Avdeev也, D.I.Kazakov?̊ and I.N.Kondrashuk円
}

\author{
Laboratory of Theoretical Physics \\ Joint Institute for Nuclear Research \\ 141980 Dubna (Moscow Region) \\ Russian Federation
}

\begin{abstract}
We investigate the possibility of generalizing differential renormalization of D.Z.Freedman, K.Johnson and J.I.Latorre in an invariant fashion to theories with infrared divergencies via an infrared $\tilde{R}$ operation. Two-dimensional $\sigma$ models and the four-dimensional $\phi^{4}$ theory diagrams with exceptional momenta are used as examples, while dimensional renormalization serves as a test scheme for comparison. We write the basic differential identities of the method simultaneously in co-ordinate and momentum space, introducing two scales which remove ultraviolet and infrared singularities. The consistent set of Fourier-transformation formulae is derived. However, the values for tadpole-type Feynman integrals in higher orders of perturbation theory prove to be ambiguous, depending on the order of evaluation of the subgraphs. In two dimensions, even earlier than this ambiguity manifests itself, renormalization-group calculations based on infrared extension of differential renormalization lead to incorrect results. We conclude that the extended differential renormalization procedure does not perform the infrared $\tilde{R}$ operation in a self-consistent way, as the original recipe does the ultraviolet $R$ operation.
\end{abstract}

\footnotetext{
${ }^{1}$ E-mail: avdeevL@theor.jinrc.dubna.su

${ }^{2}$ E-mail: kazakovD@theor.jinrc.dubna.su

${ }^{3}$ E-mail: ikond@theor.jinrc.dubna.su
} 


\section{Introduction}

Differential renormalization [1] can successfully perform an ultraviolet $R$ operation by means of replacing essentially singular quantities in co-ordinate space with derivatives of less singular expressions, and after that, by integrating the derivatives by parts in order to turn them into powers of external momenta. Then, at first sight, one sees no serious obstacles - it would just seem very natural indeed - that the same trick with singular expressions in momentum space could likewise automatically perform an infrared $\tilde{R}$ operation [2].

A need of the latter in renormalization-group calculations (leaving alone operatorproduct and other asymptotic expansions) may arise of two causes. In the first place, a quantum field theory may be intrinsically infrared-dangerous owing to the fact that the theory with zero masses may not strictly exist at all as, for example, a massless scalar field in two dimensions. However, real computations with nonzero masses are always exceedingly cumbersome technically while, on the other hand, we know that in the so-called "massless" renormalization schemes [3] (like the minimal subtraction scheme [4, 5] of dimensional or analytic regularization) there is no essential dependence of renormalization-group functions on dimensional parameters (except for a possible mixing in renormalizations of the masses themselves). Thus, we would like to set them all to zero from the very beginning but use an infrared $\tilde{R}$ operation to avoid the false infrared singularities.

In the second case, artificial infrared divergencies may be caused by a singular choice of external momenta of a Green's function, quite well defined in a general situation. For technical reasons, such a choice may seem more convenient, or it may even be the only available choice that would allow us to compute a complicated multiloop vertex-type graph analytically by means of an infrared rearrangement [6].

Although there are no theoretical grounds, like the causality principle of the local quantum field theory, for attributing a physical meaning to the infrared $\tilde{R}$ operation in itself - the reasons look quite technical both with intrinsic and artificial infrared divergencies - however, in many practically important cases its use may be crucial for achieving computational results [7, 8].

Introducing the infrared $\tilde{R}$ operation into the formalism of differential renormalization, we should keep track of three important points. First of all, the scheme should retain its self-consistency: the results should never depend on the order of manipulations we perform. The second requirement is that we should distinguish two dimensional scales: a new infrared renormalization scale $\nu$, and an ultraviolet scale $\mu$ already present in the formalism. Otherwise, infrared logarithms would be the same as ultraviolet logarithms, and we would never be able to extract from the finite renormalized Feynman diagrams the correct information about renormalization-group functions, contained in their $\mu$ dependence. And last but by no means least point is the invariance of the regularization [9], which ensures the fact that formal symmetries of a quantum field theory are retained after renormalization [5, 10].

We formulate a natural generalization of differential renormalization to theories where, besides usual ultraviolet singularities, intrinsic or artificial infrared divergencies do appear. Our particular attention is paid to two-dimensional $\sigma$ models and to the 
four-dimensional $\phi^{4}$ theory. Throughout our renormalization-group calculations, we compare the results of the extended differential renormalization with the minimal subtraction scheme of dimensional regularization as an example of an undoubtedly valid, though different, renormalization scheme in the range of scheme arbitrariness.

\section{$2 \quad \mathrm{D}=2$ : Intrinsic infrared divergencies}

As the first example, we consider two-dimensional $\sigma$ models [11, 12] where we meet infrared difficulties of both intrinsic and artificial origin.

The starting point of differential renormalization in a two-dimensional theory is the following replacement which effectively subtracts an infinite local ultraviolet counterterm proportional to the $\delta$ function in co-ordinate space:

$$
1 / x^{2}=\frac{1}{8} \square_{x} \ln ^{2}\left(x^{2} M^{2}\right),
$$

where $\square_{x}$ denotes $\left(\partial / \partial x_{\mu}\right)^{2}$ (we work in Euclidean space). The other way of looking at the matter is to consider eq.(1) as a real equality under a regularization, bearing in mind that the regularized expressions are renormalized afterwards by minimal subtractions which simply turn the regulator $M \rightarrow \infty$ into a finite renormalization scale.

With the aid of eq.(1), integrating the derivatives by parts and abandoning surface terms (which is one more prescription of the regularization), we can derive a basic set of formulae for Fourier transformations. We start with the ultraviolet-divergent integrals of the form

$$
I_{n}=\int \mathrm{d}^{2} x \mathrm{e}^{\mathrm{i} p x} \frac{\ln ^{n}\left(x^{2} M^{2}\right)}{x^{2}} .
$$

Replacing $1 / x^{2}$ through eq.(11), we integrate one $\partial / \partial x_{\mu}$ by parts. Of course, we suppose the Leibniz differentiation rule to remain valid under the regularization. The first derivative of the logarithm is a nonsingular function in two dimensions, and hence, it can be substituted explicitly as $2 x_{\mu} / x^{2}$ without any possible $\delta$-function-like terms. The resulting equation can be solved with respect to $I_{n}$ :

$$
I_{n}=-\frac{\mathrm{i} p_{\mu}}{2(n+1)} \int \mathrm{d}^{2} x \mathrm{e}^{\mathrm{i} p x} \frac{x_{\mu}}{x^{2}} \ln ^{n+1}\left(x^{2} M^{2}\right)
$$

The integral on the right-hand side has no dangerous singularities, and we can unambiguously evaluate it, using an intermediate analytic regularization which is taken off in the end,

$$
\begin{aligned}
\int \mathrm{d}^{2} x & \mathrm{e}^{\mathrm{i} p x} \frac{x_{\mu}}{x^{2}} \ln ^{n}\left(x^{2} M^{2}\right) \\
& =\left[(-)^{n} \frac{\mathrm{d}^{n}}{\mathrm{~d} \alpha^{n}} \frac{1}{\mathrm{i}} \frac{\partial}{\partial p_{\mu}} \frac{1}{\left(M^{2}\right)^{\alpha}} \int \mathrm{d}^{2} x \frac{\mathrm{e}^{\mathrm{i} p x}}{\left(x^{2}\right)^{1+\alpha}}\right]_{\alpha=0} \\
& =\left.2 \pi \mathrm{i} \frac{p_{\mu}}{p^{2}}(-)^{n} \frac{\mathrm{d}^{n}}{\mathrm{~d} \alpha^{n}}\left[\left(p^{2} / \mu^{2}\right)^{\alpha} F(\alpha)\right]\right|_{\alpha=0}
\end{aligned}
$$




$$
\begin{aligned}
& =2 \pi \mathrm{i} \frac{p_{\mu}}{p^{2}}(-)^{n} \sum_{m=0}^{n}\left(\begin{array}{c}
n \\
m
\end{array}\right) F_{n-m} \ln ^{m}\left(p^{2} / \mu^{2}\right), \\
I_{n} & =\pi \frac{(-)^{n+1}}{n+1} \sum_{m=0}^{n+1}\left(\begin{array}{c}
n+1 \\
m
\end{array}\right) F_{n+1-m} \ln ^{m}\left(p^{2} / \mu^{2}\right),
\end{aligned}
$$

where $\mu^{2}=4 M^{2} \mathrm{e}^{-2 C}$,

$$
F(\alpha)=\mathrm{e}^{-2 C \alpha} \frac{\Gamma(1-\alpha)}{\Gamma(1+\alpha)}=\exp \left[2 \sum_{m=1}^{\infty} \frac{\zeta(2 m+1)}{2 m+1} \alpha^{2 m+1}\right],
$$

$F_{m}$ are $m$ th derivatives of that function at zero,

$$
\begin{gathered}
F_{0}=1, \quad F_{1}=F_{2}=0, \quad F_{3}=4 \zeta(3), \quad F_{4}=0, \quad F_{5}=48 \zeta(5), \\
F_{6}=160 \zeta^{2}(3), \quad F_{7}=1440 \zeta(7), \quad \ldots,
\end{gathered}
$$

$\zeta(m)$ is the Riemann zeta function, and $C$ is the Euler constant.

Since the initial integrals $I_{n}$, eq.(2), were logarithmically divergent, in the most general situation we could add to the renormalized expressions (4) some constants $C_{n}$, reflecting a local arbitrariness. In the context of differential renormalization, we could leave an explicit $\delta$-function term in eq.(四). That would simply be equivalent to redefining the renormalization scale $M$. Therefore, we have chosen to absorb the arbitrariness into the definition of $M$, and no more $C_{n}$ could ever appear with the minimal subtractions on eq.(田).

Now, we can try to execute precisely the same procedure for infrared rather than ultraviolet divergencies, starting from momentum space with an infrared regulator mass $\nu \rightarrow 0$, which becomes another finite renormalization scale after the minimal subtractions:

$$
\begin{gathered}
1 / p^{2}=\frac{1}{8} \square_{p} \ln ^{2}\left(p^{2} / \nu^{2}\right) \\
\int \mathrm{d}^{2} p \mathrm{e}^{-\mathrm{i} p x} \frac{p_{\mu}}{p^{2}} \ln ^{n}\left(p^{2} / \nu^{2}\right)=-2 \pi \mathrm{i} \frac{x_{\mu}}{x^{2}}(-)^{n} \sum_{m=0}^{n}\left(\begin{array}{c}
n \\
m
\end{array}\right) F_{n-m} \ln ^{m}\left(x^{2} N^{2}\right), \\
i_{n}=\int \mathrm{d}^{2} p \mathrm{e}^{-\mathrm{i} p x} \frac{\ln ^{n}\left(p^{2} / \nu^{2}\right)}{p^{2}}=\pi \frac{(-)^{n+1}}{n+1} \sum_{m=0}^{n+1}\left(\begin{array}{c}
n+1 \\
m
\end{array}\right) F_{n+1-m} \ln ^{m}\left(x^{2} N^{2}\right) .
\end{gathered}
$$

Again, we have denoted $N^{2}=\frac{1}{4} \nu^{2} \mathrm{e}^{2 C}$. (Everywhere, $M^{2}$ and $N^{2}$ accompany $x^{2}$, while $p^{2}$ is divided by $\mu^{2}$ or $\nu^{2}$ ).

\subsection{The simplest example}

The fact that there really is an infrared difficulty for differential renormalization in two dimensions is quite obvious from considering the simplest one-loop tadpole diagram which appears in $\sigma$ models (analyzed below in subsects.2.4 and 2.5):

$$
\bigcap=\int \mathrm{d}^{2} p \frac{1}{p^{2}}
$$


The original method of ref.[1] would simply set this diagram to zero, leaving no trace of any ultraviolet logarithm. Therefore, in order to find a correct contribution to the $\beta$ function, we need a special procedure for defining such integrals which involve no external momenta or co-ordinates and are both ultraviolet- and infrared-divergent.

Let us start with the outline of our ideas in application to this integral as a simple example. We always assume that the whole scheme can be treated as an invariant regularization procedure which allows formal manipulations with divergent integrals. Then, we can identically transform eq.(9) to introduce an auxiliary external momentum argument $k_{\mu}$ :

$$
\int \mathrm{d}^{2} p \frac{1}{p^{2}}=k_{\mu} \int \mathrm{d}^{2} p \frac{1}{p^{2}} \frac{(k-p)_{\mu}}{(k-p)^{2}}-\int \mathrm{d}^{2} p \frac{p_{\mu}}{p^{2}} \frac{(k-p)_{\mu}}{(k-p)^{2}} .
$$

The first integral on the right-hand side of eq.(10) is infrared-divergent but ultravioletregular, while the second integral has only an ultraviolet singularity. Now we use the Fourier transformation to rewrite eq.(10) as

$$
\int \frac{\mathrm{d}^{2} x}{(2 \pi)^{2}} \mathrm{e}^{\mathrm{i} k x}\left(k_{\mu} \int \mathrm{d}^{2} p \frac{\mathrm{e}^{-\mathrm{i} p x}}{p^{2}}-\int \mathrm{d}^{2} p \mathrm{e}^{-\mathrm{i} p x} \frac{p_{\mu}}{p^{2}}\right) \int \mathrm{d}^{2} q \mathrm{e}^{-\mathrm{i} q x} \frac{q_{\mu}}{q^{2}} .
$$

One Fourier integral is regular [it is the simplest particular case of eq.(7)]

$$
\int \mathrm{d}^{2} p \mathrm{e}^{-\mathrm{i} p x} \frac{p_{\mu}}{p^{2}}=-2 \pi \mathrm{i} \frac{x_{\mu}}{x^{2}}
$$

The other Fourier integral in eq.(11) requires an infrared renormalization. It is $i_{0}$, eq.(8),

$$
\int \mathrm{d}^{2} p \frac{\mathrm{e}^{-\mathrm{i} p x}}{p^{2}}=-\pi \ln \left(x^{2} N^{2}\right)
$$

In the extended differential renormalization, the latter equation is derived from the momentum-space differential identity (6) via integration by parts and abandoning surface terms which are absorbed into local $p$-space counterterms in the formalism of the infrared $\tilde{R}$ operation [2]. The result involves a new infrared renormalization scale. For the case of the Fourier transform of a free scalar-field propagator (13), the physical meaning of the $\tilde{R}$ operation can easily be understood: it recovers the most essential (logarithmic) part of the massive propagator that remains as the mass goes to zero

$$
\int \mathrm{d}^{2} p \frac{\mathrm{e}^{-\mathrm{i} p x}}{p^{2}+m^{2}}=2 \pi K_{0}(m|x|)=-\pi \ln \left(\frac{1}{4} m^{2} x^{2}\right)+o(1), \quad m \rightarrow 0 .
$$

In this interpretation, the infrared differential regularization parameter in eq.(6) is proportional to the mass $\nu^{2}=m^{2} \mathrm{e}^{-2 C}$. However, the general $\tilde{R}$ formalism is not reduced to introducing a mass. It renormalizes any infrared-divergent diagram in a dual way to the ordinary $R$ operation with local $x$-space counterterms, the structure being completely analogous, but with the $x \leftrightarrow p$ interchange. 
To finish the computation of the tadpole diagram (9), we substitute eqs.(12) and (13) into eq.(11). The integrals over $x$ are taken by the original rules of differential renormalization, which lead to eqs.(3) and (41), in particular,

$$
\int \mathrm{d}^{2} x \mathrm{e}^{\mathrm{i} k x} \frac{x_{\mu}}{x^{2}} \ln \left(x^{2} N^{2}\right)=-2 \pi \mathrm{i} \frac{k_{\mu}}{k^{2}} \ln \frac{k^{2}}{\nu^{2}}, \quad \int \mathrm{d}^{2} x \frac{\mathrm{e}^{\mathrm{i} k x}}{x^{2}}=-\pi \ln \frac{k^{2}}{\mu^{2}},
$$

where the ultraviolet scale $\mu$ has appeared. In the resulting expression for the diagram (9) the dependence on the auxiliary momentum cancels, and we obtain $\pi \ln \left(\mu^{2} / \nu^{2}\right)$. Differentiating this with respect to $\ln \mu^{2}$, we reproduce the correct contribution to the $\beta$ function.

A systematic study of the implications of the infrared differential renormalization is presented below. First, in subsect.2.2, we try to derive a maximum set of relations (including singular functions) from the requirements of invariance of regularization. Since the maximum set entails a contradiction (subsect.2.3), we restrict ourselves to avoid any explicit singular differetiation. However, even without that, we recover all the essential consequences, so that the more cautious approach turns to be equivalent to the unrestricted one.

\subsection{The consistent set of Fourier integrals}

Having added the momentum-space differential identity (6), we ought to check the consistency of the scheme as a whole. We expect of a good regularization that it is invariant [9], that is, permits us to do with our integrals some formal manipulations, which is important for retaining symmetries of quantum field models. One of those features is the possibility of differentiating the integrals with respect to external parameters, for example, the Fourier-transformed argument, to compute other integrals, with external Lorentz indices, as we have done in deriving eq.(3). Other natural requirements are the possibilities of changing the order of integrations, of shifting integration variables, and of identically transforming subintegral expressions, like expanding $(p-q)^{2}$ or canceling the numerator with the denominator. Such transformations are often performed, in particular, when one does superfield algebra in supersymmetric models to reduce supergraphs to usual Feynman integrals.

Believing that the extended differential regularization can be treated as an invariant overall regularization, let us apply $-\square_{x}$ to $i_{n}$ to obtain, after canceling the denominator $p^{2}$ in eq.(8), new integrals

$$
j_{n}=-\square_{x} i_{n}=\int \mathrm{d}^{2} p \mathrm{e}^{-\mathrm{i} p x} \ln ^{n}\left(p^{2} / \nu^{2}\right),
$$

which contain no infrared divergencies, as differs from eq.(8). Note also that any arbitrary renormalization-specific constants $c_{n}$ added to $i_{n}$ are annihilated by differentiation, so that $j_{n}$ will in any case be determined uniquely.

At $n=0$, we must evidently get $j_{0}=4 \pi^{2} \delta_{2}(x)$. On the other hand, differentiating the right-hand side of eq.(8) for $i_{0}$, produces the laplacian of the logarithm. Hence,

$$
\square_{x} \ln \left(x^{2} N^{2}\right)=4 \pi \delta_{2}(x)
$$


Of course, in fact, eq.(15) is true without any reference to differential regularization $(N$ drops out, too). The identity can as well be derived by some other means, for example, by explicitly introducing the causal rule of passing over $x^{2}=0$, expanding the left-hand side of eq.(15), and directly evaluating the integral of it. The extended differential regularization reproduces the result correctly.

Now we proceed to $n=1$. The laplacian applied to the logarithm squared in $i_{1}$, eq.(8), gives us two terms: when both derivatives act on one logarithm, we use eq.(15), while the nonsingular first derivatives of the logarithm can be substituted directly. Thus, we get

$$
j_{1}=-\square_{x} i_{1}=-4 \pi^{2} \delta_{2}(x) \ln \left(x^{2} N^{2}\right)-4 \pi / x^{2} .
$$

To attach a meaning to eq.(16), we need to extend the definition of the logarithm to zero point in co-ordinate space. However, our set of formulae already includes such a definition: if we expand, on the right-hand side of eq.(1), the action of the laplacian, we get besides $1 / x^{2}$ an additional term which equals $\pi \delta_{2}(x) \ln \left(x^{2} M^{2}\right)$ in view of eq. (15). We have decided that by definition of $M$ this local term is absent in eq.(11); therefore,

$$
\delta_{2}(x) \ln x^{2}=\delta_{2}(x) \ln \left(1 / M^{2}\right)
$$

It is this formula that shows us explicitly what the regularization really changes. For the rest, everything looks as if nothing has been regularized at all, provided we manage to take proper care of singular functions.

As soon as we have established eqs.(17) and (15), we can uniformly obtain all the integrals $j_{n}$, eq.(14), from $i_{n}$, eq.(8), arriving at the general formula

$$
\begin{gathered}
j_{n}=\int \mathrm{d}^{2} p \mathrm{e}^{-\mathrm{i} p x} \ln ^{n}\left(p^{2} / \nu^{2}\right)=4 \pi(-)^{n}\left[\pi \delta_{2}(x) \sum_{m=0}^{n}\left(\begin{array}{c}
n \\
m
\end{array}\right) F_{n-m} \ln ^{m}\left(N^{2} / M^{2}\right)\right. \\
\left.+\frac{n}{x^{2}} \sum_{m=0}^{n-1}\left(\begin{array}{c}
n-1 \\
m
\end{array}\right) F_{n-1-m} \ln ^{m}\left(x^{2} N^{2}\right)\right]
\end{gathered}
$$

Quite analogously, acting with $-\square_{p}$ on eq.(4) and remembering eq.(6), we deduce the $x \leftrightarrow p$ complementary formulae

$$
\begin{gathered}
\square_{p} \ln \left(p^{2} / \mu^{2}\right)=4 \pi \delta_{2}(p) \\
\delta_{2}(p) \ln p^{2}=\delta_{2}(p) \ln \nu^{2} \\
J_{n}=\int \mathrm{d}^{2} x \mathrm{e}^{\mathrm{i} p x} \ln ^{n}\left(x^{2} M^{2}\right)=4 \pi(-)^{n}\left[\pi \delta_{2}(p) \sum_{m=0}^{n}\left(\begin{array}{c}
n \\
m
\end{array}\right) F_{n-m} \ln ^{m}\left(\nu^{2} / \mu^{2}\right)\right. \\
\left.+\frac{n}{p^{2}} \sum_{m=0}^{n-1}\left(\begin{array}{c}
n-1 \\
m
\end{array}\right) F_{n-1-m} \ln ^{m}\left(p^{2} / \mu^{2}\right)\right] .
\end{gathered}
$$

Evidently, the ratios $N^{2} / M^{2}$ in eq.(18) and $\nu^{2} / \mu^{2}$ in eq. (20) are the same.

Another observation is that, as $J_{n}$ is ultraviolet regular, it does not generate an ultraviolet scale parameter by itself. Therefore, $M$ in eq.(20) is just a dummy argument 
(strictly related to $\mu$ on the right-hand side) while the real stamp of the regularization on $J_{n}$ is the infrared scale $\nu$ from eq.(19). The self-consistency of replacing $M$ in eq.(20) can be checked directly by re-expanding the logarithm with an arbitrary mass argument $\Lambda$ by the Newton's binomial, and resumming the right-hand side. The result

$$
\begin{gathered}
\int \mathrm{d}^{2} x \mathrm{e}^{\mathrm{i} p x} \ln ^{n}\left(x^{2} \Lambda^{2}\right)=4 \pi(-)^{n}\left[\pi \delta_{2}(p) \sum_{m=0}^{n}\left(\begin{array}{c}
n \\
m
\end{array}\right) F_{n-m} \ln ^{m}\left(\nu^{2} / \lambda^{2}\right)\right. \\
\left.+\frac{n}{p^{2}} \sum_{m=0}^{n-1}\left(\begin{array}{c}
n-1 \\
m
\end{array}\right) F_{n-1-m} \ln ^{m}\left(p^{2} / \lambda^{2}\right)\right]
\end{gathered}
$$

actually looks the same as eq.(20). Likewise, we can freely change $\nu$ in eq.(18) to a dummy variable $\lambda$ which can even be set equal to $\mu$,

$$
\begin{gathered}
\int \mathrm{d}^{2} p \mathrm{e}^{-\mathrm{i} p x} \ln ^{n}\left(p^{2} / \lambda^{2}\right)=4 \pi(-)^{n}\left[\pi \delta_{2}(x) \sum_{m=0}^{n}\left(\begin{array}{c}
n \\
m
\end{array}\right) F_{n-m} \ln ^{m}\left(\Lambda^{2} / M^{2}\right)\right. \\
\left.+\frac{n}{x^{2}} \sum_{m=0}^{n-1}\left(\begin{array}{c}
n-1 \\
m
\end{array}\right) F_{n-1-m} \ln ^{m}\left(x^{2} \Lambda^{2}\right)\right] .
\end{gathered}
$$

The intermediate integrals with Lorentz indices, eqs.(3) and (7), are both ultravioletand infrared-regular. Thus, they introduce no scales at all, $\mu$ and $\nu$ being their dummy arguments.

The next step in checking the consistency is to take the Fourier transforms of eqs.(21) and (22). One easily sees that the resulting formulae allow us to recursively recover the very first integrals: $I_{n}$, eq.(21), and $i_{n}$, eq.(8). The double circle closes. By induction, using binomial re-expansions, we can prove that formulae (4) and (8) are exactly reproduced without any $C_{n}$ and $c_{n}$, which reflects the fact that we made eqs.(17) and (19) agree with the differential relations (11) and (6). For other masses under the logarithms, we get quite definite additive terms,

$$
\begin{aligned}
\int \mathrm{d}^{2} x & \mathrm{e}^{\mathrm{i} p x} \frac{\ln ^{n}\left(x^{2} \Lambda^{2}\right)}{x^{2}} \\
= & \frac{\pi}{n+1}\left[(-)^{n+1} \sum_{m=0}^{n+1}\left(\begin{array}{c}
n+1 \\
m
\end{array}\right) F_{n+1-m} \ln ^{m}\left(p^{2} / \lambda^{2}\right)-\ln ^{n+1}\left(\lambda^{2} / \mu^{2}\right)\right], \\
\int \mathrm{d}^{2} p & \mathrm{e}^{-\mathrm{i} p x} \frac{\ln ^{n}\left(p^{2} / \lambda^{2}\right)}{p^{2}} \\
= & \frac{\pi}{n+1}\left[(-)^{n+1} \sum_{m=0}^{n+1}\left(\begin{array}{c}
n+1 \\
m
\end{array}\right) F_{n+1-m} \ln ^{m}\left(x^{2} \Lambda^{2}\right)-\ln ^{n+1}\left(\nu^{2} / \lambda^{2}\right)\right],
\end{aligned}
$$

with $\lambda^{2}=4 \Lambda^{2} \mathrm{e}^{-2 C}$ in eqs.(21)-(24). 


\subsection{Ambiguities in repeated integrals}

Everything looks fine at the moment, and we could start to compute diagrams. However, the point is that we actually need a little more than just switching to the Fourier transforms and back. Namely, we expect that a property similar to associativity of convolution will hold. Suppose, there are three quantities

$$
a_{n}(p)=\int \mathrm{d}^{2} x \mathrm{e}^{\mathrm{i} p x} A_{n}(x), \quad A_{n}(x)=\int \frac{d^{2} p}{(2 \pi)^{2}} \mathrm{e}^{-\mathrm{i} p x} a_{n}(p),
$$

$(n=1,2,3)$, and we are going to evaluate the integral of their product in momentum space. We can represent it in three different ways, substituting eq.(25) either for $a_{1}$, $a_{2}$, or $a_{3}$ :

$$
\begin{aligned}
X & =\int \mathrm{d}^{2} p a_{1}(p) a_{2}(p) a_{3}(p) \\
& =\int \mathrm{d}^{2} x A_{1}(x) \int \mathrm{d}^{2} p \mathrm{e}^{-\mathrm{i} p x} a_{2}(p) a_{3}(p) \\
& =\int \mathrm{d}^{2} x A_{2}(x) \int \mathrm{d}^{2} p \mathrm{e}^{-\mathrm{i} p x} a_{1}(p) a_{3}(p) \\
& =\int \mathrm{d}^{2} x A_{3}(x) \int \mathrm{d}^{2} p \mathrm{e}^{-\mathrm{i} p x} a_{1}(p) a_{2}(p),
\end{aligned}
$$

where the integral over $x$ can be evaluated by a trick of inserting a unit

$$
\int \mathrm{d}^{2} x \ldots=\int \mathrm{d}^{2} q \delta_{2}(q) \int \mathrm{d}^{2} x \mathrm{e}^{\mathrm{i} q x} \ldots
$$

and subsequently applying eq.(19). Either way (26)-(28) should lead to the same result in the framework of a consistent regularization. Let us, however, substitute particular expressions

$$
a_{1}(p)=\frac{2 \ln \left(p^{2} / \nu^{2}\right)}{p^{2}}, \quad a_{2}=a_{3}=\ln \left(p^{2} / \mu^{2}\right) .
$$

All the integrals that arise can be evaluated with the aid of eqs.(21)-(24), after products of different logarithms are re-expanded in powers of one of them. The results are

$$
\begin{aligned}
& X \stackrel{(26)}{\Rightarrow} \pi\left[\frac{1}{6} \ln ^{4}\left(\nu^{2} / \mu^{2}\right)+\frac{8}{3} \zeta(3) \ln \left(\nu^{2} / \mu^{2}\right)\right], \\
& X \stackrel{\text { 277, (28) }}{\Longrightarrow} \pi\left[\frac{1}{6} \ln ^{4}\left(\nu^{2} / \mu^{2}\right)-\frac{4}{3} \zeta(3) \ln \left(\nu^{2} / \mu^{2}\right)\right] .
\end{aligned}
$$

We see that there are two ways of computing the integral, which give us two different answers. The resulting expressions (31) and (32) coincide only if we set $\nu^{2}=\mu^{2}$, that is, use the same scale for renormalizing both ultraviolet and infrared divergencies. As has been said at the beginning, this would not be satisfactory for renormalization-group applications.

One may suspect that the inconsistency has resulted from undue liberty in our dealing with singular functions. Indeed, in establishing our set of formulae, we exceeded 
the necessary minimum of operations, essential for evaluating Feynman integrals. So, in principle, we can do without explicit differentiation the result of which might involve a singular function.

Then, we ought to exclude all the singular relations, (15), (16), (17), and (19), thus, being unable to get $j_{n}$, eq.(14), from $i_{n}$. Nevertheless, the basic divergent integrals themselves $I_{n}$ and $i_{n}$, eqs.(壮) and (8), can be derived from the differential identities eqs.(1) and (6) through intermediate finite integrals, eqs.(7) and (3), as before. Reexpanding the logarithms, we reproduce the integrals with arbitrary mass arguments, eqs.(23) and (24); and finally, eqs.(22) and (21) are obtained as their resummed Fourier transforms with the particular cases of eqs.(18) and (20).

Although the trick of eq.(29) becomes of no use, we can, all the same, define the general external-parameter-independent integrals in eqs.(26)-(28) by utilizing the assumed invariance of the regularization:

$$
\begin{aligned}
\int \mathrm{d}^{2} x & \frac{\ln ^{n}\left(x^{2} \Lambda^{2}\right)}{x^{2}}=\int \mathrm{d}^{2} x \frac{(y-x) \cdot y-(y-x) \cdot x}{(y-x)^{2} x^{2}} \ln ^{n}\left(x^{2} \Lambda^{2}\right) \\
= & \int \frac{\mathrm{d}^{2} p}{(2 \pi)^{2}} \mathrm{e}^{-\mathrm{i} p y}\left(\int \mathrm{d}^{2} z \mathrm{e}^{\mathrm{i} p z} \frac{z_{\mu}}{z^{2}}\right)\left[\int \mathrm{d}^{2} x \mathrm{e}^{\mathrm{i} p x}\left(\frac{y_{\mu}}{x^{2}}-\frac{x_{\mu}}{x^{2}}\right) \ln ^{n}\left(x^{2} \Lambda^{2}\right)\right] \\
= & \frac{\pi}{n+1}\left[(-)^{n+1} \sum_{m=0}^{n+1}\left(\begin{array}{c}
n+1 \\
m
\end{array}\right) F_{n+1-m} \ln ^{m}\left(N^{2} / \Lambda^{2}\right)-\ln ^{n+1}\left(\Lambda^{2} / M^{2}\right)\right],
\end{aligned}
$$

where we have made use of eqs.(3), (23) and (24). Note that the term with $m=0$ is always present, even at $\Lambda^{2}=N^{2}$. In the same way, we can evaluate the corresponding momentum integrals

$$
\begin{aligned}
\int \mathrm{d}^{2} p & \frac{\ln ^{n}\left(p^{2} / \lambda^{2}\right)}{p^{2}} \\
& =\frac{\pi}{n+1}\left[(-)^{n+1} \sum_{m=0}^{n+1}\left(\begin{array}{c}
n+1 \\
m
\end{array}\right) F_{n+1-m} \ln ^{m}\left(\lambda^{2} / \mu^{2}\right)-\ln ^{n+1}\left(\nu^{2} / \lambda^{2}\right)\right] .
\end{aligned}
$$

Now, for the chosen $a_{1}, a_{2}$, and $a_{3}$, as in eq.(30), the integrals on the right-hand sides of eqs.(26)-(28) are directly calculable, and we reproduce the two different results (31) and (32) again.

\section{$2.4 \mathrm{~N}=2$ supersymmetric sigma model}

Yet, let us see how grave this contradiction is from the practical viewpoint of computing Feynman diagrams. We consider first the four-loop approximation in the $N=2$ supersymmetric two-dimensional $\sigma$ model. After the supergraph algebra in the background-field formalism is done [12], there appears just one nontrivial divergent four-loop momentum integral which is presented in fig.1 1a. The rest of the divergent integrals are of the primitive tadpole type (fig.1 $\mathrm{b}$ ); we leave them alone for the moment. 

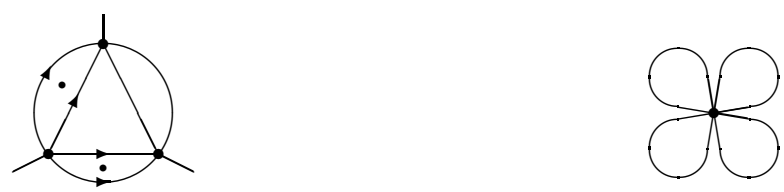

(a)

(b)

Figure 1: The divergent four-loop momentum integrals in the $N=2$ supersymmetric two-dimensional $\sigma$ model. Lines represent $1 / p^{2}$ propagators, arrows stand for additional $p_{\mu}$ in the numerator, and small dots denote contractions of Lorentz indices.

The model involves intrinsic infrared divergencies due to zero masses on the lines without components of momenta in numerators. Besides, there may appear artificial infrared divergencies if some of the external momenta in fig. 17a are set to zero. In fact, for technical reasons, we simply cannot do without this, to reduce the problem to successively evaluating one-loop propagator-type graphs. The three external lines of the diagram correspond to identical operator structures of the background field. Therefore, nullifying one of the three momenta, we obtain two different graphs shown in fig.2, while setting all of them to zero, we get a pure tadpole. The diagram of fig.2 $2 \mathrm{~b}$ and the complicated tadpole graph contain artificial infrared divergencies.

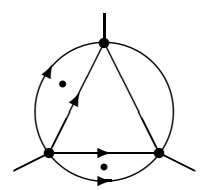

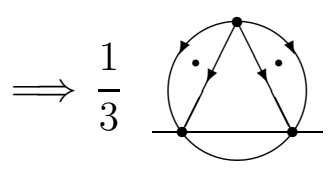

(a)

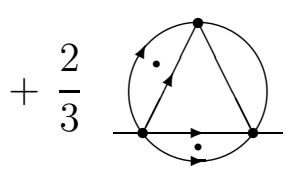

(b)

Figure 2: Two possibilities that arise when one of the external momenta is set to zero.

We can easily compute the two one-loop subgraphs both in co-ordinate and in momentum space (25) by means of eqs.(17), (23), (24), and (21):

$$
\begin{aligned}
& 0 \bigcirc x \Longrightarrow-\frac{1}{4 \pi^{2}} \frac{1}{x^{2}}, \\
& 0 . \because \frac{p}{(4 \pi)^{2}} \ln ^{2}\left(x^{2} N^{2}\right),
\end{aligned}
$$

Now, the diagram of fig. Za can be calculated as follows. We first compute its two-loop propagator-type subgraph in momentum space as the square of the one-loop expression (35). Then, we transform the subgraph to co-ordinate space

$$
0 . x \frac{\mathrm{d}^{2} p}{4 \pi^{2}} \mathrm{e}^{-\mathrm{i} p x}\left[\frac{\ln \left(p^{2} / \mu^{2}\right)}{4 \pi}\right]^{2}=\frac{1}{\pi(4 \pi)^{2}} \frac{2 \ln \left(x^{2} M^{2}\right)}{x^{2}}
$$

The whole diagram is just a product of eq.(37) and the infrared one-loop subgraph (36) in $x$ space. Re-expanding the infrared logarithm squared in terms of $\ln \left(x^{2} M^{2}\right)$, and switching back to momentum space by means of eq.(四), we arrive at the result 


$$
\begin{gathered}
\text { fig.2a } \Rightarrow \frac{1}{(4 \pi)^{4}[} \frac{1}{2} \ln ^{4}\left(p^{2} / \mu^{2}\right)-\frac{4}{3} \ln ^{3}\left(p^{2} / \mu^{2}\right) \ln \left(\nu^{2} / \mu^{2}\right)+\ln ^{2}\left(p^{2} / \mu^{2}\right) \ln ^{2}\left(\nu^{2} / \mu^{2}\right) \\
\left.+8 \zeta(3) \ln \left(p^{2} / \mu^{2}\right)-\frac{16}{3} \zeta(3) \ln \left(\nu^{2} / \mu^{2}\right)\right] .
\end{gathered}
$$

The same result is obtained if we leave the one-loop subgraph as it is, but reduce eq.(37) to $\ln \left(x^{2} N^{2}\right)$, take the Fourier transform of the product via eq.(23), and finally, re-expand it in the ultraviolet logarithms.

In this way, using only the Fourier transformation formulae, we can compute any diagram of recursively one-loop propagator structure. In particular, we consecutively evaluate

$$
\begin{gathered}
\Rightarrow x \Longrightarrow \int \frac{\mathrm{d}^{2} p}{4 \pi^{2}} \mathrm{e}^{-\mathrm{i} p x} \frac{\ln \left(p^{2} / \mu^{2}\right)}{4 \pi} \frac{1}{4 \pi} \frac{2 \ln \left(p^{2} / \nu^{2}\right)}{p^{2}} \\
=\frac{1}{(4 \pi)^{3}}\left[\frac{2}{3} \ln ^{3}\left(x^{2} M^{2}\right)+\ln ^{2}\left(x^{2} M^{2}\right) \ln \left(\nu^{2} / \mu^{2}\right)-\frac{1}{3} \ln ^{3}\left(\nu^{2} / \mu^{2}\right)+\frac{8}{3} \zeta(3)\right], \\
\text { fig.27b } \Rightarrow \frac{1}{(4 \pi)^{4}}\left[\frac{1}{6} \ln ^{4}\left(p^{2} / \mu^{2}\right)-\frac{1}{3} \ln ^{3}\left(p^{2} / \mu^{2}\right) \ln \left(\nu^{2} / \mu^{2}\right)+\frac{1}{3} \ln \left(p^{2} / \mu^{2}\right) \ln ^{3}\left(\nu^{2} / \mu^{2}\right)\right. \\
\left.-\frac{4}{3} \zeta(3) \ln \left(\nu^{2} / \mu^{2}\right)\right] .
\end{gathered}
$$

The answer for the tadpole diagram at zero momenta in fig. 1. proves to be ambiguous. If we first compute its infrared-regular two-loop subgraph (37), and then evaluate the tadpole by the $\delta$-function trick (29) or by eq.(33), we get

$$
\Longrightarrow \frac{1}{(4 \pi)^{4}}\left[\frac{1}{6} \ln ^{4}\left(\nu^{2} / \mu^{2}\right)+\frac{8}{3} \zeta(3) \ln \left(\nu^{2} / \mu^{2}\right)\right]
$$

like in eq.(31). The use of the direct momentum-space formula (34) leads to the same answer. On the other hand, starting from the infrared-divergent two-loop subgraph (39), we repeat eq.(32):

$$
\Longrightarrow \frac{1}{(4 \pi)^{4}}\left[\frac{1}{6} \ln ^{4}\left(\nu^{2} / \mu^{2}\right)-\frac{4}{3} \zeta(3) \ln \left(\nu^{2} / \mu^{2}\right)\right]
$$

Both answers (41) and (42) can be obtained from eqs.(48) and (40), respectively, by replacing $p^{2}$ with $\nu^{2}$.

To estimate the meaning of the results, it is worth comparing them with the unconditionally consistent scheme of dimensional renormalization. The infrared $\tilde{R}$ and ultraviolet $R$ operation ought to be done explicitly then. Without entering into details, let us present as an example (fig. 3) the structure of renormalizations for the diagram of fig.2. $\mathrm{b}$. 

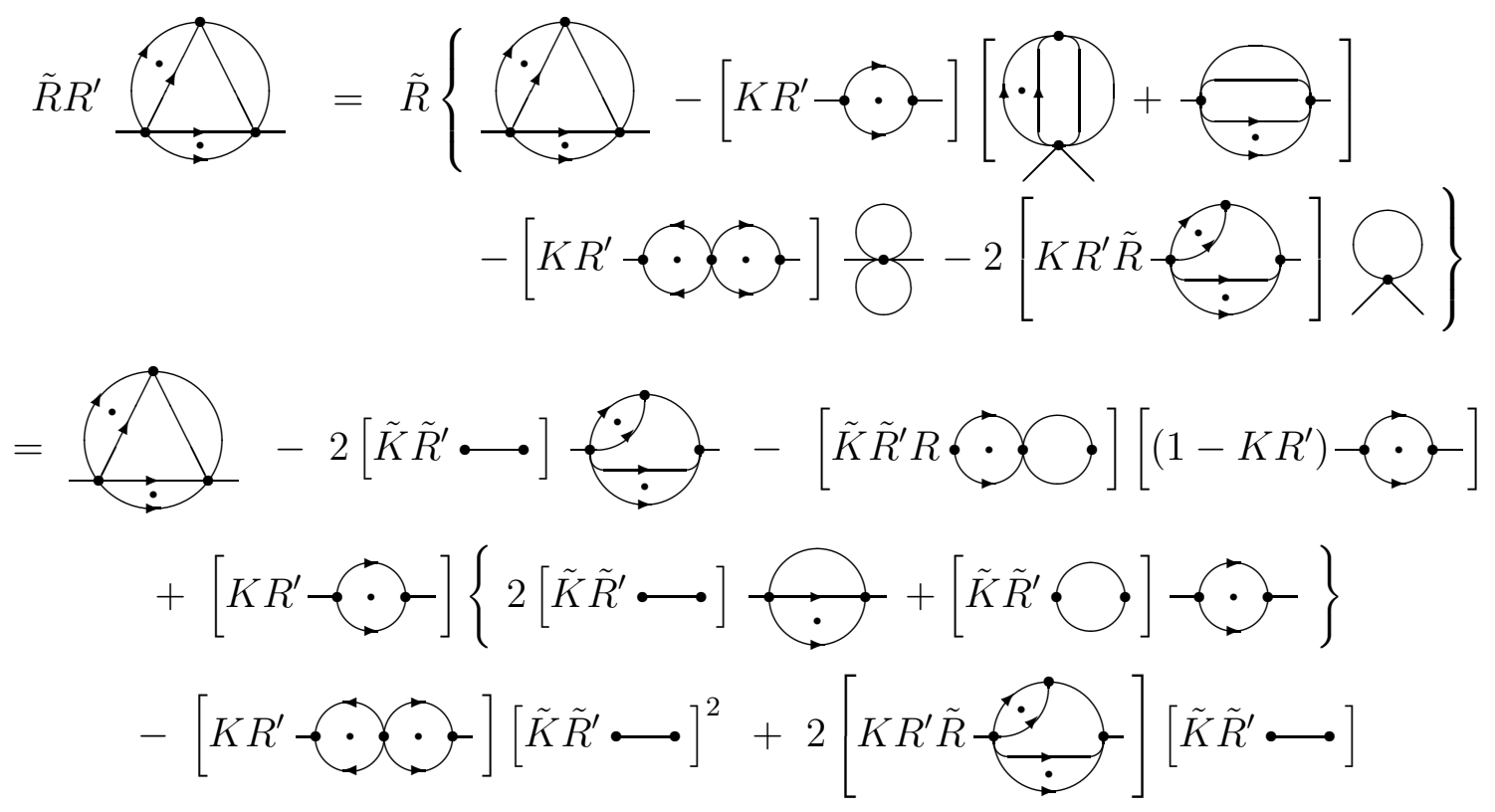

Figure 3: The structure of the incomplete ultraviolet $R^{\prime}$ operation and the full infrared $\tilde{R}$ operation. A subtracted ultraviolet subgraph is shrunk to a point, while an infrared subgraph is just deleted. After the $\tilde{R}$, we have omitted zero tadpole graphs.

The subtraction operators $K$ and $\tilde{K}$ pick out poles in $\varepsilon=(2-D) / 2$ in momentum and co-ordinate space, respectively. We keep different ultraviolet and infrared renormalization scales. Thus, every momentum loop integration is, as usual, accompanied by a $\left(\mu^{2}\right)^{\varepsilon}$ factor, while the proper dimension of infrared counterterms proportional to $\delta_{2-2 \varepsilon}(p)$ is restored by additional denominators of $\left(\nu^{2}\right)^{\varepsilon}$. For example, in fig. 3 we should substitute

$$
\tilde{K} \tilde{R}^{\prime} R \circlearrowleft=\frac{1}{3 \varepsilon^{3}}\left(\mu^{2} / \nu^{2}\right)^{3 \varepsilon},
$$

one $\left(\mu^{2}\right)^{\varepsilon}$ coming from the integration annihilated by the $\delta$ function.

Instead of doing the infrared $\tilde{R}$ operation in the diagram language, as in fig. 3 , it may technically be easier to subtract singular momentum-space expressions directly by means of a simple formula [8]

$$
\frac{1}{\left(p^{2}\right)^{1+n \varepsilon}} \stackrel{\tilde{R}}{\rightarrow} \frac{1}{\left(p^{2}\right)^{1+n \varepsilon}}+\pi \frac{\left(\nu^{2}\right)^{-(n+1) \varepsilon}}{(n+1) \varepsilon} \delta_{2-2 \varepsilon}(p),
$$

leaving only pole terms on subtracting complicated subgraphs with a prefactor.

Here are the results of dimensional renormalization in the so-called $G$-scheme 13]

$$
\begin{aligned}
& (4 \pi)^{4} \quad R^{\prime} \tilde{R}=-\frac{1}{6 \varepsilon^{4}}+\frac{\zeta(3)}{\varepsilon} \\
& +\frac{1}{2} \ln ^{4}\left(p^{2} / \mu^{2}\right)-\frac{4}{3} \ln ^{3}\left(p^{2} / \mu^{2}\right) \ln \left(\nu^{2} / \mu^{2}\right)+\ln ^{2}\left(p^{2} / \mu^{2}\right) \ln ^{2}\left(\nu^{2} / \mu^{2}\right) \\
& +8 \zeta(3) \ln \left(p^{2} / \mu^{2}\right)-\frac{28}{3} \zeta(3) \ln \left(\nu^{2} / \mu^{2}\right)+\frac{3}{2} \zeta(4)
\end{aligned}
$$


$(4 \pi)^{4} \quad R^{\prime} \tilde{R}$

$$
\begin{aligned}
& \text { - }=-\frac{1}{6 \varepsilon^{4}}+\frac{\zeta(3)}{\varepsilon}=\frac{1}{3} \ln ^{4}\left(p^{2} / \mu^{2}\right)-\left(p^{2} / \mu^{2}\right) \ln \left(\nu^{2} / \mu^{2}\right)+\frac{1}{3} \ln \left(p^{2} / \mu^{2}\right) \ln ^{3}\left(\nu^{2} / \mu^{2}\right) \\
& -\frac{4}{3} \zeta(3)\left[\ln \left(p^{2} / \mu^{2}\right)+\ln \left(\nu^{2} / \mu^{2}\right)\right]+\frac{3}{2} \zeta(4) \\
& 6 \varepsilon^{4}+\frac{\zeta(3)}{\varepsilon}+\frac{1}{6} \ln ^{4}\left(\nu^{2} / \mu^{2}\right)-4 \zeta(3) \ln \left(\nu^{2} / \mu^{2}\right) .
\end{aligned}
$$$$
(4 \pi)^{4} \quad R^{\prime} \tilde{R}
$$

Comparing eqs.(38)-(42) with eqs.(43)-(45), we come to the following conclusions. The results of the extended differential renormalization disagree with the dimensionally renormalized diagrams obtained by subtracting pole contributions, $R \tilde{R}=(1-K) R^{\prime} \tilde{R}$. Moreover, this disagreement does not fit into the framework of the scheme arbitrariness by just redefining $\mu$ and $\nu$ : eq. (44) involves a $\ln \left(p^{2} / \mu^{2}\right)$ term absent in eq. (40). Forming the weighed sum, according to fig.2, does not help in improving the agreement. The tadpole graphs are not well-defined at all, none of the results coinciding with eq.(45), neither eq.(41) nor (42).

One cannot completely avoid tadpole-graph ambiguities even at $\mu=\nu$ (this would be sufficient for asymptotic expansions). Already for the five-loop tadpole diagram shown in fig. 4 , we can get three different answers at $\mu=\nu$, starting from its one-, two-, or three-loop infrared-divergent subgraphs. This is not just an artificial example: the graph appears in the five-loop calculations of ref. $8 \|$.

$$
(4 \pi)^{5} \longrightarrow \frac{48}{5} \zeta(5), \frac{32}{5} \zeta(5), \frac{12}{5} \zeta(5) \text {. }
$$

Figure 4: The five-loop tadpole graph, the value of which in the extended differential renormalization is ambiguous even at $\mu=\nu$.

Trying to extract from eqs. 38)-(42) the contributions to the renormalization-group $\beta$ function by differentiating the expressions with respect to $\ln \mu^{2}$, we find that the result depends on $\ln \left(\nu^{2} / \mu^{2}\right)$ and $\ln \left(p^{2} / \mu^{2}\right)$. In the present case, the only nontrivial diagram of fig.1 $1 \mathrm{a}$ is proportional to a new invariant structure in the Riemann tensors and the background field, which was not present in lower orders of perturbation theory. Thus, no compensation of higher-order logarithms from lower-order graphs can occur, as it happens in ordinary renormalizable field theories. The ignored contributions from the primitive tadpole-reducible graphs of fig. [1]b cannot save the situation with higher-order logarithms of the momentum since they contain nothing but $\ln ^{4}\left(\nu^{2} / \mu^{2}\right)$. This problem is present in dimensional renormalization as well, if we attempt to find the $\beta$ function by using the finite logarithms instead of applying the traditional method [4] based on the first-order pole in $\varepsilon$. Even at $p^{2}=\nu^{2}=\mu^{2}$, fig. (2 with the finite parts of eqs.(43) and (44) does not reproduce the correct $\beta$ function [12 extracted from the pole term, although the dimensionally renormalized tadpole graph gives the consistent answer in both ways. The cause of these difficulties is non-renormalizability of the model, as a 
consequence of which the renormalization-group-like equations can only be written in terms of the pole contributions rather than directly in finite logarithms [14, 11].

\subsection{A renormalizable sigma model}

It would be natural then to consider a renormalizable model, where the renormalization-group functions can as well be computed from finite logarithms. As such a model, we can choose the $n$-component $\overrightarrow{\mathbf{n}}$ field in two dimensions,

$$
\mathcal{L}=(2 h)^{-1}\left(\partial_{\mu} \overrightarrow{\mathbf{n}}\right)^{2}, \quad \overrightarrow{\mathbf{n}}^{2}=1
$$

which can be rewritten as a special case of the simplest bosonic $\sigma$ model [11 for $n-1$ independent components

$$
\mathcal{L}=\frac{1}{2}\left(\partial_{\mu} \phi^{j}\right) g_{j k} \partial_{\mu} \phi^{k}, \quad g_{j k}=\delta_{j k}+\frac{h}{1-h \phi^{2}} \phi_{j} \phi_{k}
$$

For this particular form of the metric with the co-ordinates $\phi_{j}$, we have

$$
\begin{array}{cc}
g^{j k}=\delta^{j k}-h \phi^{j} \phi^{k}, & \Gamma_{j k}^{l}=h g_{j k} \phi^{l}, \\
R_{j k l m}=h\left(g_{j l} g_{k m}-g_{j m} g_{k l}\right), & R_{j k}=(n-2) h g_{j k} .
\end{array}
$$

The invariant charge $\bar{h}=Z^{-1} h$ is determined by the charge renormalization constant $Z$. The contributing Feynman diagrams in the background-field formalism [11] up to two loops are presented in fig.5.

$$
\begin{gathered}
-R_{j k}\left(\partial_{\mu} \phi^{j}\right) \partial_{\mu} \phi^{k} \\
+\frac{1}{12}\left(2 R_{j}^{a} R_{a k}+3 R^{a b c}{ }_{j} R_{a b c k}\right)\left(\partial_{\mu} \phi^{j}\right) \partial_{\mu} \phi^{k} \\
-\frac{1}{6} R_{j}{ }^{a b}{ }_{k} R_{a b}\left(\partial_{\mu} \phi^{j}\right) \partial_{\mu} \phi^{k} \\
+\frac{4}{9} R_{j}{ }^{(a b) c} R_{k a b c}\left(\partial_{\mu} \phi^{j}\right) \partial_{\nu} \phi^{k} \\
+\frac{8}{9} R_{j}{ }^{(a b) c} R_{k(c b) a}\left(\partial_{\mu} \phi^{j}\right) \partial_{\nu} \phi^{k}
\end{gathered}
$$

Figure 5: The one- and two-loop corrections to the effective action of the twodimensional bosonic $\sigma$ model without torsion. The contributions to $Z$ are obtained by normalizing to the tree term $\frac{1}{2}\left(\partial_{\mu} \phi^{j}\right) g_{j k} \partial_{\mu} \phi^{k}$.

The results of dimensional renormalization for the $\overrightarrow{\mathbf{n}}$ field are

$$
R^{\prime} \tilde{R}(\text { fig. } 5 \mathrm{a})=-(n-2) g_{j k}\left(\partial_{\mu} \phi^{j}\right)\left(\partial_{\mu} \phi^{k}\right) \frac{h}{4 \pi}\left[\frac{1}{\varepsilon}+\ln \frac{\mu^{2}}{\nu^{2}}\right]
$$




$$
\begin{gathered}
R^{\prime} \tilde{R}(\text { fig. } 5 \mathrm{~b})=\frac{1}{6}(n+1)(n-2) g_{j k}\left(\partial_{\mu} \phi^{j}\right)\left(\partial_{\mu} \phi^{k}\right) \frac{h^{2}}{(4 \pi)^{2}}\left[-\frac{1}{\varepsilon^{2}}+\ln ^{2} \frac{\mu^{2}}{\nu^{2}}\right] \\
R^{\prime} \tilde{R}\left(\text { fig. }[\mathrm{b})=\frac{1}{6}(n-2)^{2} g_{j k}\left(\partial_{\mu} \phi^{j}\right)\left(\partial_{\mu} \phi^{k}\right) \frac{h^{2}}{(4 \pi)^{2}}\left[\frac{1}{\varepsilon^{2}}-\ln ^{2} \frac{\mu^{2}}{\nu^{2}}\right]\right. \\
R^{\prime} \tilde{R}(\text { fig. } 5 \mathrm{~d})=\frac{2}{3}(n-2) g_{j k}\left(\partial_{\mu} \phi^{j}\right)\left(\partial_{\nu} \phi^{k}\right) \frac{h^{2}}{(4 \pi)^{2}}\left[\frac { g _ { \mu \nu } } { 2 } \left(\frac{1}{\varepsilon^{2}}-\frac{1}{\varepsilon}\right.\right. \\
\left.\left.+\ln 2 \frac{p^{2}}{\nu^{2}}-2 \ln \frac{p^{2}}{\nu^{2}}-\ln \frac{\mu^{2}}{\nu^{2}}-2 \ln \frac{\mu^{2}}{\nu^{2}}+1\right)-\frac{p_{\mu} p_{\nu}}{p^{2}}\left(\ln ^{2} \frac{p^{2}}{\nu^{2}}-2 \ln \frac{p^{2}}{\nu^{2}}+2\right)\right] \\
R^{\prime} \tilde{R}(\text { fig. } 5 \mathrm{e})=-\frac{2}{3}(n-2) g_{j k}\left(\partial_{\mu} \phi^{j}\right)\left(\partial_{\nu} \phi^{k}\right) \frac{h^{2}}{(4 \pi)^{2}}\left[-\frac{g_{\mu \nu}}{4}\left(\frac{1}{\varepsilon^{2}}-\frac{1}{\varepsilon}\right.\right. \\
\left.\left.+\ln ^{2} \frac{p^{2}}{\nu^{2}}-2 \ln \frac{p^{2}}{\nu^{2}}-\ln ^{2} \frac{\mu^{2}}{\nu^{2}}-2 \ln \frac{\mu^{2}}{\nu^{2}}+1\right)-\frac{p_{\mu} p_{\nu}}{p^{2}}\left(\ln \frac{p^{2}}{\nu^{2}}-1\right)\right]
\end{gathered}
$$

These formulae lead to the renormalized

$$
Z_{\text {dim }}=1-(n-2)\left(2 \ln \frac{\mu^{2}}{\nu^{2}}\right) \frac{h}{4 \pi}-(n-2)\left(\frac{1}{3} \ln ^{2} \frac{p^{2}}{\nu^{2}}+2 \ln \frac{\mu^{2}}{p^{2}}+3\right) \frac{h^{2}}{(4 \pi)^{2}} .
$$

Independence of the invariant charge on $\mu^{2}$ leads then to the following expression for the $\beta$ function

$$
\beta_{\operatorname{dim}}(h)=h\left(\frac{\partial Z}{\partial \ln \mu^{2}}\right)\left[\left(1-h \frac{\partial}{\partial h}\right) Z\right]^{-1}=-2(n-2) \frac{h^{2}}{4 \pi}\left(1+\frac{h}{4 \pi}\right) .
$$

The result is the same as obtained from the $1 / \varepsilon$-pole terms in eqs.(46)-(50). It also agrees with the known $\beta$ function for the $\overrightarrow{\mathbf{n}}$-field $O(n)$ sigma model [15] where no infrared $\tilde{R}$ operation was ever used.

To be capable of evaluating the Feynman integrals of fig.5(d,e) in the extended differential renormalization, we need to extend the set of formulae to a more general kind of expressions. The new differential relation is

$$
\frac{x_{\mu}}{\left(x^{2}\right)^{2}} \ln ^{n}\left(x^{2} M^{2}\right)=-\frac{1}{2} n ! \frac{\partial}{\partial x_{\mu}}\left[\frac{1}{x^{2}} \sum_{m=0}^{n} \frac{\ln ^{m}\left(x^{2} M^{2}\right)}{m !}+\pi \delta_{2}(x)\right] .
$$

The scale parameter $M$ is the same as in eq.(1), while the coefficient of the $\delta$ function is uniquely fixed by consistency with the previously established formulae. We multiply eq.(52) by $x_{\mu}$ and take the Fourier transform. After integrating the derivative by parts on the right-hand side, we get the integrals of the known types, eqs.(2) and (3), while the left-hand side becomes just $I_{n}$, eq.(田). Thus, we can easily find the necessary coefficient. 
The Feynman integrals in the extended differential renormalization, to be compared with the expressions in square brackets in eqs. 46)-(50), can now be evaluated to

$$
\begin{aligned}
\text { fig.5a } \Rightarrow & \ln \left(\mu^{2} / \nu^{2}\right) \\
\text { fig.5]b } \mathrm{b} & \ln ^{2}\left(\mu^{2} / \nu^{2}\right), \\
\text { fig.5 } \mathrm{c} \Rightarrow & -\ln ^{2}\left(\mu^{2} / \nu^{2}\right), \\
\text { fig.5]d } \Rightarrow & \frac{1}{2} g_{\mu \nu}\left[\ln ^{2}\left(p^{2} / \nu^{2}\right)-2 \ln \left(p^{2} / \nu^{2}\right)-\ln ^{2}\left(\mu^{2} / \nu^{2}\right)+2\right] \\
& -\left(p_{\mu} p_{\nu} / p^{2}\right)\left[\ln ^{2}\left(p^{2} / \nu^{2}\right)-2 \ln \left(p^{2} / \nu^{2}\right)+2\right], \\
\text { fig.5] } \Rightarrow & -\frac{1}{4} g_{\mu \nu}\left[\ln ^{2}\left(p^{2} / \nu^{2}\right)-2 \ln \left(p^{2} / \nu^{2}\right)-\ln ^{2}\left(\mu^{2} / \nu^{2}\right)+2\right] \\
& -\left(p_{\mu} p_{\nu} / p^{2}\right)\left[\ln \left(p^{2} / \nu^{2}\right)-1\right] .
\end{aligned}
$$

We see that eqs.(56) and (57) differ from the finite parts of eqs.(49) and (50) in dimensional renormalization by the lack of the lower-degree logarithm of $\mu^{2}$. As a result, we get quite a different charge renormalization

$$
\begin{gathered}
Z_{\text {diff }}=1-(n-2)\left(2 \ln \frac{\mu^{2}}{\nu^{2}}\right) \frac{h}{4 \pi}+(n-2)\left(-\frac{1}{3} \ln ^{2} \frac{p^{2}}{\nu^{2}}+2 \ln \frac{p^{2}}{\nu^{2}}-2\right) \frac{h^{2}}{(4 \pi)^{2}}, \\
\beta_{\text {diff }}(h)=-2(n-2) h^{2} /(4 \pi)+0 \cdot h^{3} .
\end{gathered}
$$

Equations (58) and (51) disagree beyond the range allowed by the scheme arbitrariness in perturbation theory because in a one-charge model the two-loop $\beta$ function must be scheme-independent [16].

Thus, the infrared extension of differential renormalization completely fails in twodimensional models with intrinsic infrared divergencies. The results of multiloop renormalization-group calculations may prove to be incorrect or ill-defined. In higher orders of perturbation theory, ambiguities cannot be avoided even if we use the same scale $\mu=\nu$ for renormalizing infrared and ultraviolet divergencies.

\section{$3 \quad \mathrm{D}=4:$ Artificial infrared divergences}

There are no off-shell infrared divergencies in $D=4$. However, they may appear as a result of the infrared rearrangement in Feynman diagrams, aimed to the simplification of multiloop calculations [6], without which some complicated diagrams may technically occur unattainable for analytic computation. Indeed, the usual trick is to put some (or all) external momenta to be zero (that is to integrate over the corresponding co-ordinates in $x$ space), and then to calculate divergencies, for example, the poles in dimensional regularization. According to the theorem proven in ref.[5], after subtraction of subdivergencies, the singular part of a diagram is a polynomial in external momenta and internal masses, which is reduced to a constant independent of them in the case of a logarithmic divergency. This allows one to simplify calculations, taking care of the infrared divergencies that may appear as a result of nullifying some momenta. These artificial infrared divergencies can be subtracted by means of the infrared $\tilde{R}$ operation [2]. 
Our aim here is to check whether the formalism of differential renormalization can be extended to perform the infrared $\tilde{R}$ operation in the same way as it performs the usual ultraviolet $R$ operation in a renormalizable model.

To be concrete, consider the massless scalar field theory $\phi_{(D=4)}^{4}$. The basic equation of differential renormalization [1] is the four-dimensional analog of eq.(1),

$$
1 /\left(x^{2}\right)^{2}=-\frac{1}{4} \square_{x}\left[\ln \left(x^{2} M^{2}\right) / x^{2}\right],
$$

and its counterpart in momentum space is

$$
1 /\left(p^{2}\right)^{2}=-\frac{1}{4} \square_{p}\left[\ln \left(p^{2} / \nu^{2}\right) / p^{2}\right] .
$$

Here, $M$ and $\nu$ are the ultraviolet and infrared scales, respectively.

Following the rules of the differential renormalization method [1], we perform the calculations, replacing all the singular expressions according to eqs.(59) and (60), and then integrating the derivatives by parts and ignoring surface terms. However, we should point out an important difference from our line of reasoning with the set of Fourier-transformation formulae in two dimensions. If we allow us to differentiate Feynman integrals, generating singular expressions, then the circle of checking consistency of the integrals does not close by a nonzero additive constant. Thus, we are forced to follow a more cautious way, avoiding any explicit differentiation, the result of which may be singular. Neither shall we attribute any meaning to the logarithm at its singular zero point.

Obeying these rules and using an intermediate analytic regularization when needed, we get the following set of formulae:

$$
\begin{aligned}
I_{n} & =\int \mathrm{e}^{\mathrm{i} p x} \mathrm{~d}^{4} x \frac{\ln ^{n}\left(x^{2} M^{2}\right)}{x^{2}}=\left.4 \pi^{2} \frac{(-)^{n}}{p^{2}} \frac{\mathrm{d}^{n}}{d \alpha^{n}}\left[\left(p^{2} / \mu^{2}\right)^{\alpha} F(\alpha)\right]\right|_{\alpha=0} \\
i_{n} & =\int \mathrm{e}^{-\mathrm{i} p x} \mathrm{~d}^{4} p \frac{\ln ^{n}\left(p^{2} / \nu^{2}\right)}{p^{2}}=\left.4 \pi^{2} \frac{(-)^{n}}{x^{2}} \frac{\mathrm{d}^{n}}{d \alpha^{n}}\left[\left(x^{2} N^{2}\right)^{\alpha} F(\alpha)\right]\right|_{\alpha=0} \\
J_{n} & =\int \mathrm{e}^{\mathrm{i} p x} \mathrm{~d}^{4} x \frac{\ln ^{n}\left(x^{2} M^{2}\right)}{\left(x^{2}\right)^{2}}=\pi^{2} \frac{(-)^{n+1}(n-1) !}{n+1} \times \\
& \times\left.\sum_{k=1}^{n} \frac{1}{(k-1) !}\left\{\frac{\mathrm{d}^{k+1}}{d \alpha^{k+1}}\left[\left(\frac{p^{2}}{\mu^{2}}\right)^{\alpha} F(\alpha)\right]-2 k \frac{\mathrm{d}^{k}}{d \alpha^{k}}\left[\left(\frac{p^{2}}{\mu^{2}}\right)^{\alpha} \frac{F(\alpha)}{1+\alpha}\right]\right\}\right|_{\alpha=0} \\
j_{n} & =\int \mathrm{e}^{-\mathrm{i} p x} \mathrm{~d}^{4} p \frac{\ln ^{n}\left(p^{2} / \nu^{2}\right)}{\left(p^{2}\right)^{2}}=\pi^{2} \frac{(-)^{n+1}(n-1) !}{n+1} \times \\
& \times\left.\sum_{k=1}^{n} \frac{1}{(k-1) !}\left\{\frac{\mathrm{d}^{k+1}}{d \alpha^{k+1}}\left[\left(x^{2} N^{2}\right)^{\alpha} F(\alpha)\right]-2 k \frac{\mathrm{d}^{k}}{d \alpha^{k}}\left[\left(x^{2} N^{2}\right)^{\alpha} \frac{F(\alpha)}{1+\alpha}\right]\right\}\right|_{\alpha=0}
\end{aligned}
$$

where $F(\alpha)$ is the same as in eq.(5) above. In particular, to the lowest orders, one gets

$$
I_{0}=4 \pi^{2} / p^{2}
$$




$$
\begin{aligned}
& I_{1}=-\left(4 \pi^{2} / p^{2}\right) \ln \left(p^{2} / \mu^{2}\right) \\
& I_{2}=\left(4 \pi^{2} / p^{2}\right) \ln ^{2}\left(p^{2} / \mu^{2}\right) \\
& J_{0}=-\pi^{2} \ln \left(p^{2} / \mu^{2}\right) \\
& J_{1}=\pi^{2}\left[\frac{1}{2} \ln ^{2}\left(p^{2} / \mu^{2}\right)-\ln \left(p^{2} / \mu^{2}\right)+1\right] \\
& J_{2}=\pi^{2}\left[-\frac{1}{3} \ln ^{3}\left(p^{2} / \mu^{2}\right)+\ln ^{2}\left(p^{2} / \mu^{2}\right)-2 \ln \left(p^{2} / \mu^{2}\right)-\frac{4}{3} \zeta(3)+2\right]
\end{aligned}
$$

and the same for the space-time integrals $i_{n}$ and $j_{n}$ with the interchange $x^{2} \leftrightarrow p^{2}$, $M^{2} \leftrightarrow 1 / \nu^{2}, \mu^{2} \leftrightarrow 1 / N^{2}$. The relation between $\mu$ and $M$ remains the same as in two dimensions; capitals refer to $x$ space. Using eqs.(59)-(64), (65)-(70), we can perform all the calculations.

In principle, the set of formulae can be supplemented with renormalization-scaleindependent singular equations

$$
\square_{x}\left(1 / x^{2}\right)=-16 \pi^{2} \delta_{4}(x), \quad \square_{p}\left(1 / p^{2}\right)=-16 \pi^{2} \delta_{4}(p) .
$$

However, integrating a derivative by parts, we can avoid using them explicitly, for the sake of safety always keeping ourselves at least one step off the singular threshold.

Consider, for pedagogical purposes, the vertex function in the two-loop approximation (see fig.6).

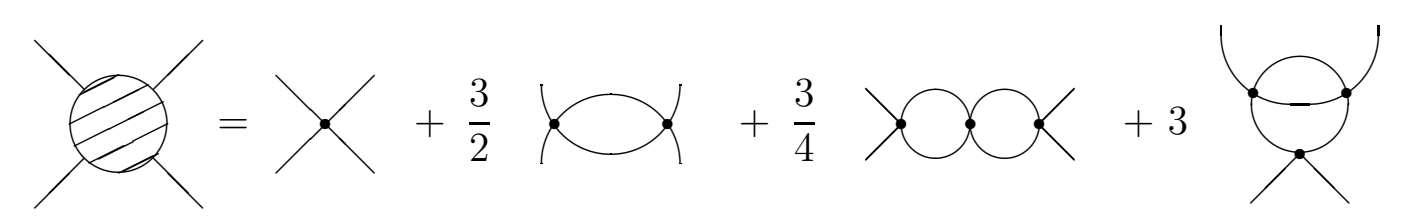

Figure 6: The vertex function of the $\phi^{4}$ theory in the two-loop approximation.

We demonstrate the infrared peculiarities of the extension of differential renormalization by the calculation of the renormalization-group $\beta$ function. It coincides with the anomalous dimension of four-point vertex up to the two-loop propagator contribution ignored hereafter.

\subsection{Non-exceptional momenta}

To simplify the calculation, we reduce the diagrams to the propagator type by nullifying some external momenta. One can do this in two different ways. The first way is to take non-exceptional external momenta without creating any infrared divergencies. Then, according to eqs.(59)-(69), we have [Hereafter, we ignore obvious factors of $\pi^{2}$ coming from the integrals, together with $(2 \pi)^{-4}$ from the loops, so that the contribution of each diagram should be divided by $\left(16 \pi^{2}\right)^{n}$, where $n$ is the number of loops.]

$$
p \rightarrow \widehat{x}_{0}=\int \mathrm{d}^{4} x \frac{\mathrm{e}^{\mathrm{i} p x}}{\left(x^{2}\right)^{2}}=-\ln \left(p^{2} / \mu^{2}\right)
$$




$$
\begin{aligned}
p \rightarrow \mathrm{d}_{x} & =\int \frac{\mathrm{d}^{4} x \mathrm{~d}^{4} y \mathrm{e}^{\mathrm{i} p x}}{\left(y^{2}\right)^{2}\left[(y-x)^{2}\right]^{2}}=\left[\int \mathrm{d}^{4} x \frac{\mathrm{e}^{\mathrm{i} p x}}{\left(x^{2}\right)^{2}}\right]^{2}=\ln ^{2}\left(p^{2} / \mu^{2}\right), \\
& =\int \frac{\mathrm{d}^{4} x \mathrm{~d}^{4} y \mathrm{e}^{\mathrm{i} p x}}{x^{2}(y-x)^{2}\left(y^{2}\right)^{2}} \\
& =\int \mathrm{d}^{4} x \frac{\mathrm{e}^{\mathrm{i} p x}}{x^{2}} \frac{\ln \left(x^{2} M^{2}\right)}{x^{2}}=\frac{1}{(y-x)^{2}} \frac{-\square_{y}}{4}\left[\frac{\ln \left(y^{2} M^{2}\right)}{y^{2}}\right]
\end{aligned}
$$

Summing up all these contributions, we get the invariant charge as follows:

$$
\bar{h}_{\text {diff }}=h+\frac{3}{2} h^{2} \ln \left(p^{2} / \mu^{2}\right)+h^{3}\left[\frac{9}{4} \ln ^{2}\left(p^{2} / \mu^{2}\right)-3 \ln \left(p^{2} / \mu^{2}\right)+3\right] .
$$

The $\beta$ function is defined by

$$
\beta(h)=\mu^{2} \frac{\mathrm{d} h}{\mathrm{~d} \mu^{2}}
$$

while the invariant charge $\bar{h}$ is $\mu$-independent. Differentiating eq.(71) with respect to $\mu^{2}$, we get

$$
\beta(h)=\frac{3}{2} h^{2}-3 h^{3} .
$$

Compare this calculation with the one that uses dimensional regularization and the $\overline{\mathrm{MS}}$ scheme. One has

$$
\begin{aligned}
& \Longrightarrow=\int \frac{\mathrm{d}^{4-2 \varepsilon} x \mathrm{e}^{\mathrm{i} p x}}{\left(x^{2}\right)^{2-2 \varepsilon}}=\frac{1}{\varepsilon(1-2 \varepsilon)}\left(\frac{\mu^{2}}{p^{2}}\right)^{\varepsilon} \\
& K R^{\prime}=1 / \varepsilon, \quad R=-\ln \left(p^{2} / \mu^{2}\right)+2 ; \\
& \bigcirc=\int \frac{\mathrm{d}^{4-2 \varepsilon} x \mathrm{~d}^{4-2 \varepsilon} y \mathrm{e}^{\mathrm{i} p x}}{\left(y^{2}\right)^{2-2 \varepsilon}(y-x)^{2(2-2 \varepsilon)}}=\left[\frac{\left(\mu^{2} / p^{2}\right)^{\varepsilon}}{\varepsilon(1-2 \varepsilon)}\right]^{2} \text {, } \\
& R^{\prime}=\left[\frac{1}{\varepsilon(1-2 \varepsilon)}\left(\frac{\mu^{2}}{p^{2}}\right)^{\varepsilon}\right]^{2}-\frac{2}{\varepsilon}\left[\frac{1}{\varepsilon(1-2 \varepsilon)}\left(\frac{\mu^{2}}{p^{2}}\right)^{\varepsilon}\right] \\
& K R^{\prime}=-1 / \varepsilon^{2}, \quad R=\ln ^{2}\left(p^{2} / \mu^{2}\right)-4 \ln \left(p^{2} / \mu^{2}\right)+4 ; \\
& \mathcal{C}-\int \frac{\mathrm{d}^{4-2 \varepsilon} x \mathrm{~d}^{4-2 \varepsilon} y \mathrm{e}^{\mathrm{i} p x}}{\left(y^{2}\right)^{2-2 \varepsilon}\left[(y-x)^{2}\right]^{1-\varepsilon}\left(x^{2}\right)^{1-\varepsilon}}=\int \frac{\mathrm{d}^{4-2 \varepsilon} x \mathrm{e}^{\mathrm{i} p x}}{\left(x^{2}\right)^{1-\varepsilon}} \frac{1}{\varepsilon(1-2 \varepsilon)\left(x^{2}\right)^{1-2 \varepsilon}},
\end{aligned}
$$




$$
\begin{aligned}
R^{\prime} & =\int \frac{\mathrm{d}^{4-2 \varepsilon} x \mathrm{e}^{\mathrm{i} p x}}{\left(x^{2}\right)^{1-\varepsilon}}\left[\frac{1}{\varepsilon(1-2 \varepsilon)\left(x^{2}\right)^{1-2 \varepsilon}}-\frac{1}{\varepsilon\left(x^{2}\right)^{1-\varepsilon}}\right] \\
& =\frac{\left(\mu^{2} / p^{2}\right)^{2 \varepsilon}}{2 \varepsilon^{2}(1-2 \varepsilon)(1-3 \varepsilon)}-\frac{\left(\mu^{2} / p^{2}\right)^{2 \varepsilon}}{\varepsilon^{2}(1-2 \varepsilon)}, \\
K R^{\prime} & =-\frac{1}{2 \varepsilon^{2}}+\frac{1}{2 \varepsilon}, \quad R=\frac{1}{2} \ln ^{2}\left(p^{2} / \mu^{2}\right)-3 \ln \left(p^{2} / \mu^{2}\right)+\frac{11}{2} .
\end{aligned}
$$

Thus, for the bare and invariant charges, we have, respectively,

$$
h_{\mathrm{B}}=\left(\mu^{2}\right)^{\varepsilon}\left[h+\frac{3}{2}\left(h^{2}-h^{3}\right) / \varepsilon+\frac{9}{4} h^{3} / \varepsilon^{2}\right] \text {, }
$$

and

$$
\bar{h}_{\overline{\mathrm{MS}}}=h+\frac{3}{2} h^{2}\left[\ln \left(p^{2} / \mu^{2}\right)-2\right]+h^{3}\left[\frac{9}{4} \ln ^{2}\left(p^{2} / \mu^{2}\right)-12 \ln \left(p^{2} / \mu^{2}\right)+\frac{39}{2}\right] .
$$

We can now calculate the $\beta$ function, differentiating either eq.(74) or eq.(75) with respect to $\mu^{2}$ and taking into account eq.(72). Both ways lead to eq.(73) for the $\beta$ function.

\subsection{Exceptional momenta in differential renormalization}

The second possibility is to take some exceptional momenta. In our case, setting two of them equal to zero, we get the following decomposition (see fig.7).

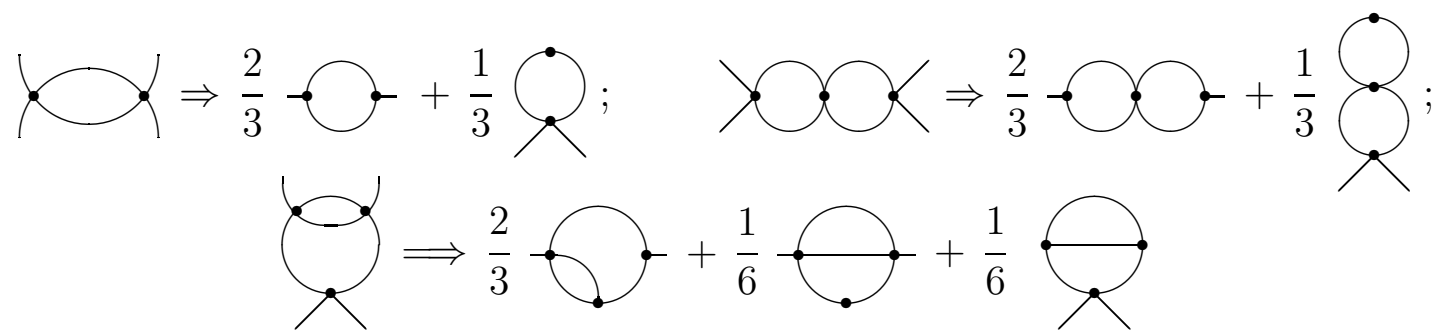

Figure 7: Two-loop vertex diagrams in case of exceptional momenta.

Now, we ought to consistently remove infrared divergencies by defining the artificial tadpole graphs which are both ultraviolet- and infrared-divergent. We can use the invariance of the regularization, to get a definition,

$$
\zeta=\int \frac{\mathrm{d}^{4} x}{\left(x^{2}\right)^{2}} \stackrel{\text { def }}{=} \int \frac{\mathrm{d}^{4} x}{\left(x^{2}\right)^{2}} \frac{x^{2}-2 x y+y^{2}}{(x-y)^{2}}=-\ln \left(\nu^{2} / \mu^{2}\right)-2,
$$

where we have evaluated the integrals, according to eqs.(59)-(70). 
Consider now the two-loop diagrams. We have

$$
\begin{aligned}
3 & =\int \frac{\mathrm{d}^{4} x}{\left(x^{2}\right)^{2}} \int \frac{\mathrm{d}^{4} y}{\left[(x-y)^{2}\right]^{2}}=\left[\ln \left(\nu^{2} / \mu^{2}\right)+2\right]^{2} \\
& =\int \mathrm{d}^{4} x \frac{\mathrm{e}^{\mathrm{i} p x}}{\left(x^{2}\right)^{2}} \int \frac{\mathrm{d}^{4} y}{y^{2}(y-x)^{2}}=\int \mathrm{d}^{4} x \frac{\mathrm{e}^{\mathrm{i} p x}}{\left(x^{2}\right)^{2}} \int \frac{\mathrm{d}^{4} q}{\left(q^{2}\right)^{2}} \mathrm{e}^{-\mathrm{i} q x} \\
& =-\int \mathrm{d}^{4} x \frac{\mathrm{e}^{\mathrm{i} p x}}{\left(x^{2}\right)^{2}} \ln \left(x^{2} N^{2}\right)=-\int \mathrm{d}^{4} x \frac{\mathrm{e}^{\mathrm{i} p x}}{\left(x^{2}\right)^{2}}\left[\ln \left(x^{2} M^{2}\right)+\ln \left(\nu^{2} / \mu^{2}\right)\right] \\
& =-\frac{1}{2} \ln ^{2}\left(p^{2} / \mu^{2}\right)+\ln \left(p^{2} / \mu^{2}\right) \ln \left(\nu^{2} / \mu^{2}\right)+\ln \left(p^{2} / \mu^{2}\right)-1 .
\end{aligned}
$$

As for the last diagram of fig.7, it can be calculated in two different ways: first, integrating over $y$ in a usual way, and then considering the $x$ tadpole-type integral; or conversely. Proceeding in both ways, we get, respectively,

$$
\begin{aligned}
& \mathrm{C}_{x} y=\int \frac{\mathrm{d}^{4} x \mathrm{~d}^{4} y}{\left(y^{2}\right)^{2}(y-x)^{2} x^{2}}=\int \mathrm{d}^{4} x \frac{\ln \left(x^{2} M^{2}\right)}{\left(x^{2}\right)^{2}} \\
& =\int \mathrm{d}^{4} x \frac{\ln \left(x^{2} M^{2}\right)}{\left(x^{2}\right)^{2}} \frac{x^{2}-2 x z+z^{2}}{(x-z)^{2}}=\frac{1}{2} \ln ^{2}\left(\nu^{2} / \mu^{2}\right)+\ln \left(\nu^{2} / \mu^{2}\right), \\
& =-\int \frac{\mathrm{d}^{4} x \mathrm{~d}^{4} y}{\left(y^{2}\right)^{2}(y-x)^{2} x^{2}}=-\int \mathrm{d}^{4} y \frac{\ln \left(y^{2} N^{2}\right)}{\left(y^{2}\right)^{2}} y \frac{\ln \left(y^{2} M^{2}\right)+\ln \left(\nu^{2} / \mu^{2}\right)}{\left(y^{2}\right)^{2}}=\frac{1}{2} \ln ^{2}\left(\nu^{2} / \mu^{2}\right)+\ln \left(\nu^{2} / \mu^{2}\right) .
\end{aligned}
$$

Coincidence of these two expressions gives us, thus, a self-consistency check of the definition of the artificial tadpole graph (76).

Combining these two-loop contributions with those already found for non-exceptional momenta, we finally get the invariant charge

$$
\begin{aligned}
& \bar{h}_{\text {diff }}^{\text {(ex })}=h+h^{2}\left[\ln \left(p^{2} / \mu^{2}\right)+\frac{1}{2} \ln \left(\nu^{2} / \mu^{2}\right)+1\right]+h^{3}\left[\frac{5}{4} \ln ^{2}\left(p^{2} / \mu^{2}\right)\right. \\
& \left.+\frac{1}{2} \ln \left(p^{2} / \mu^{2}\right) \ln \left(\nu^{2} / \mu^{2}\right)+\frac{1}{2} \ln ^{2}\left(\nu^{2} / \mu^{2}\right)-\frac{3}{2} \ln \left(p^{2} / \mu^{2}\right)+\frac{3}{2} \ln \left(\nu^{2} / \mu^{2}\right)+\frac{5}{2}\right] .
\end{aligned}
$$

We see that eq.(78) differs from eq.(71). However, differentiating it with respect to $\mu^{2}$, we obtain the $\beta$ function in agreement with eq.(73).

\subsection{Exceptional momenta in dimensional renormalization}

To get a better understanding of the situation with exceptional momenta, we again compare the extended differential renormalization with dimensional regularization in 
the $\overline{\mathrm{MS}}$ scheme. In the latter case, we use the infrared $\tilde{R}$ operation to remove infrared divergencies:

$$
\begin{aligned}
& \mathcal{C}=\int \frac{\mathrm{d}^{4-2 \varepsilon} p}{\left(p^{2}\right)^{2}}, \quad \tilde{R}=\left(\mu^{2}\right)^{\varepsilon} \int \mathrm{d}^{4-2 \varepsilon} p\left[\frac{1}{\left(p^{2}\right)^{2}}+\frac{\delta_{4-2 \varepsilon}(p)}{\varepsilon\left(\nu^{2}\right)^{\varepsilon}}\right]=\frac{1}{\varepsilon}\left(\frac{\mu^{2}}{\nu^{2}}\right)^{\varepsilon}, \\
& K R^{\prime} \tilde{R}=1 / \varepsilon, \quad R^{*}=\ln \left(\mu^{2} / \nu^{2}\right) ; \\
& \zeta=\int \frac{\mathrm{d}^{4-2 \varepsilon} p}{\left(p^{2}\right)^{2}} \int \frac{\mathrm{d}^{4-2 \varepsilon} q}{\left(q^{2}\right)^{2}}, \quad \tilde{R}=\left[\frac{1}{\varepsilon}\left(\frac{\mu^{2}}{\nu^{2}}\right)^{\varepsilon}\right]^{2} \text {, } \\
& R^{\prime} \tilde{R}=\frac{1}{\varepsilon^{2}}\left(\frac{\mu^{2}}{\nu^{2}}\right)^{2 \varepsilon}-\frac{2}{\varepsilon^{2}}\left(\frac{\mu^{2}}{\nu^{2}}\right)^{\varepsilon}, K R^{\prime} \tilde{R}=-1 / \varepsilon^{2}, \quad R^{*}=\ln ^{2}\left(\mu^{2} / \nu^{2}\right) ; \\
& \tilde{R} R^{\prime} \bigcirc=\left(\mu^{2}\right)^{\varepsilon} \int \mathrm{d}^{4-2 \varepsilon} p\left[\frac{1}{\left(p^{2}\right)^{2}}+\frac{1}{\varepsilon} \frac{\delta_{4-2 \varepsilon}(p)}{\left(\nu^{2}\right)^{\varepsilon}}\right]\left[\frac{1}{\varepsilon(1-2 \varepsilon)} \frac{\left(\mu^{2}\right)^{\varepsilon}}{\left[(p-q)^{2}\right]^{\varepsilon}}-\frac{1}{\varepsilon}\right] \\
& =-\frac{\left(\mu^{2} / p^{2}\right)^{2 \varepsilon}}{2 \varepsilon^{2}(1-3 \varepsilon)}+\frac{\left(\mu^{2} / p^{2}\right)^{\varepsilon}\left(\mu^{2} / \nu^{2}\right)^{\varepsilon}}{\varepsilon^{2}(1-2 \varepsilon)}-\frac{\left(\mu^{2} / \nu^{2}\right)^{2 \varepsilon}}{\varepsilon^{2}} \text {, } \\
& K R^{\prime} \tilde{R}=-\frac{1}{2 \varepsilon^{2}}+\frac{1}{2 \varepsilon}, \quad R^{*}=-\frac{1}{2} \ln ^{2} \frac{p^{2}}{\mu^{2}}+\ln \frac{p^{2}}{\mu^{2}} \ln \frac{\nu^{2}}{\mu^{2}}+\ln \frac{p^{2}}{\mu^{2}}-2 \ln \frac{\nu^{2}}{\mu^{2}}-\frac{1}{2} ; \\
& R^{\prime} \longmapsto=\left(\mu^{2}\right)^{\varepsilon} \int \mathrm{d}^{4-2 \varepsilon} p\left[\frac{1}{\varepsilon(1-2 \varepsilon)} \frac{\left(\mu^{2}\right)^{\varepsilon}}{\left(p^{2}\right)^{2+\varepsilon}}-\frac{1}{\varepsilon} \frac{1}{\left(p^{2}\right)^{2}}\right], \\
& \tilde{R} R^{\prime}=\left(\mu^{2}\right)^{\varepsilon} \int \mathrm{d}^{4-2 \varepsilon} p\left[\frac{1}{\varepsilon(1-2 \varepsilon)} \frac{\left(\mu^{2}\right)^{\varepsilon}}{\left(p^{2}\right)^{2+\varepsilon}}+\frac{1+\varepsilon}{2 \varepsilon^{2}} \frac{\left(\mu^{2}\right)^{\varepsilon}}{\left(\nu^{2}\right)^{2 \varepsilon}} \delta_{4-2 \varepsilon}(p)\right. \\
& \left.-\frac{1}{\varepsilon} \frac{1}{\left(p^{2}\right)^{2}}-\frac{1}{\varepsilon^{2}} \frac{\delta_{4-2 \varepsilon}(p)}{\left(\nu^{2}\right)^{\varepsilon}}\right]=\frac{1+\varepsilon}{2 \varepsilon^{2}}\left(\frac{\mu^{2}}{\nu^{2}}\right)^{2 \varepsilon}-\frac{1}{\varepsilon^{2}}\left(\frac{\mu^{2}}{\nu^{2}}\right)^{\varepsilon}, \\
& K R^{\prime} \tilde{R}=-\frac{1}{2 \varepsilon^{2}}+\frac{1}{2 \varepsilon}, \quad R^{*}=\frac{1}{2} \ln ^{2}\left(\mu^{2} / \nu^{2}\right)+\ln \left(\mu^{2} / \nu^{2}\right) .
\end{aligned}
$$

Combining everything together, we get for the bare charge precisely eq.(74), and for the invariant charge

$$
\begin{aligned}
& \bar{h}_{\overline{\mathrm{MS}}}^{\text {(ex) }}=h+h^{2}\left[\ln \left(p^{2} / \mu^{2}\right)+\frac{1}{2} \ln \left(\nu^{2} / \mu^{2}\right)-2\right]+h^{3}\left[\frac{5}{4} \ln ^{2}\left(p^{2} / \mu^{2}\right)\right. \\
& \left.\quad+\frac{1}{2} \ln \left(p^{2} / \mu^{2}\right) \ln \left(\nu^{2} / \mu^{2}\right)+\frac{1}{2} \ln ^{2}\left(\nu^{2} / \mu^{2}\right)-\frac{15}{2} \ln \left(p^{2} / \mu^{2}\right)-\frac{3}{2} \ln \left(\nu^{2} / \mu^{2}\right)+\frac{51}{4}\right] .
\end{aligned}
$$

Note that eq.(79) does not coincide with eq.(78). However, if we differentiate eq.(79) with respect to $\mu^{2}$, we reproduce the correct value of the $\beta$ function eq.(73). 
Hence, in dimensional regularization, when one uses $R^{*}$ operation, the $\beta$ function calculated both from infinite and finite parts is independent of external momenta in the $\overline{\mathrm{MS}}$ scheme. This is also true for the finite expressions obtained with the aid of the extended differential renormalization, which give us the correct value of the $\beta$ function despite the fact that the finite corrections to the invariant charge eqs.(71) and (78) are different from those in the $\overline{\mathrm{MS}}$ scheme, eqs. (75) and (79). This difference is, however, trivial and reflects just the renormalization scheme arbitrariness. Moreover, to two loops, the finite part of each diagram in the extended differential renormalization can be obtained, up to a constant, from the corresponding $\overline{\mathrm{MS}}$ renormalized expression by a shift: $\ln \mu^{2} \rightarrow \ln \mu^{2}-2$. This statement happens to be independent of the presence or absence of infrared divergencies.

\subsection{Higher-order ambiguities}

This pleasant picture is, however, spoiled in higher orders. To see this, let us consider the following four-loop artificial tadpole graph:

$$
\overbrace{z}^{y}=\int \frac{\mathrm{d}^{4} x \mathrm{~d}^{4} y \mathrm{~d}^{4} z \mathrm{~d}^{4} t}{\left(y^{2}\right)^{2}(y-x)^{2}(x-z)^{2}\left[(z-t)^{2}\right]^{3} t^{2}}
$$

To evaluate the internal propagator-type subgraph, we need to add a new equation to the set of basic formulae, eqs.(59)-(70),

$$
\frac{1}{\left(x^{2}\right)^{3}}=-\frac{1}{32} \square_{x}^{2} \frac{\ln \left(x^{2} M^{2}\right)}{x^{2}}+\frac{3}{16} \pi^{2} \square_{x} \delta_{4}(x),
$$

where the coefficient of the last term is strictly fixed by the requirement of consistency with the rest of the formulae after multiplying both sides by $x^{2}$. Performing the internal integration with the aid of eq. (80), we reduce the diagram to the following integral,

$$
I=-\frac{1}{2} \int \frac{\mathrm{d}^{4} x \mathrm{~d}^{4} y\left[\ln \left(x^{2} M^{2}\right)+\frac{3}{2}\right]}{\left(y^{2}\right)^{2}(y-x)^{2} x^{2}},
$$

which, again, can be integrated first over $y$ and then over $x$, or in the opposite order. We have, respectively,

$$
\begin{aligned}
I & =-\frac{1}{2} \int \mathrm{d}^{4} x \frac{\ln \left(x^{2} M^{2}\right)\left[\ln \left(x^{2} M^{2}\right)+\frac{3}{2}\right]}{\left(x^{2}\right)^{2}} \\
& =-\frac{1}{2} \int \mathrm{d}^{4} x \frac{\ln \left(x^{2} M^{2}\right)\left[\ln \left(x^{2} M^{2}\right)+\frac{3}{2}\right]}{\left(x^{2}\right)^{2}} \frac{x^{2}-2 x z+z^{2}}{(x-z)^{2}} \\
& =\frac{1}{6} \ln ^{3}\left(\nu^{2} / \mu^{2}\right)+\frac{1}{8} \ln ^{2}\left(\nu^{2} / \mu^{2}\right)-\frac{3}{4} \ln \left(\nu^{2} / \mu^{2}\right)+\frac{2}{3} \zeta(3),
\end{aligned}
$$




$$
\begin{aligned}
I & =\frac{1}{2} \int \frac{\mathrm{d}^{4} y}{\left(y^{2}\right)^{2}} \int \frac{\mathrm{d}^{4} q}{q^{2}} \mathrm{e}^{-\mathrm{i} q y} \frac{\ln \left(q^{2} / \mu^{2}\right)-\frac{3}{2}}{q^{2}} \\
& =\frac{1}{2} \int \frac{\mathrm{d}^{4} y}{\left(y^{2}\right)^{2}}\left\{\frac{1}{2} \ln ^{2}\left(y^{2} N^{2}\right)+\left[\frac{1}{2}-\ln \left(\nu^{2} / \mu^{2}\right)\right] \ln \left(y^{2} N^{2}\right)+1\right\} \\
& =\frac{1}{6} \ln ^{3}\left(\nu^{2} / \mu^{2}\right)+\frac{1}{8} \ln ^{2}\left(\nu^{2} / \mu^{2}\right)-\frac{3}{4} \ln \left(\nu^{2} / \mu^{2}\right)-\frac{1}{3} \zeta(3)-1 .
\end{aligned}
$$

In contrast to the previous case of eqs.(77), the two expressions (82) and (82) are different. It turns out to be a general situation, which simply has not yet manifested itself at the two-loop level but becomes evident at the fourth loop [In fact, integrals like eq.(81) appear already at the three-loop level]. Thus, we see that, in $D=4$ either, the infrared extension of differential renormalization does not provide us with a selfconsistent definition of tadpole graphs. On the other hand, abandoning the infrared $\tilde{R}$ operation and just nullifying artificial tadpoles, we would not be able to make use of infrared rearrangement at all.

Therefore, we conclude that the infrared extension of differential renormalization cannot perform the infrared $\tilde{R}$ operation, like the original scheme does the ultraviolet $R$ operation.

\section{Summary}

We have examined the possibility of generalizing differential regularization in an invariant fashion to theories with infrared divergencies. Both in $D=2$ and $D=4$, the basic differential identities of the method, written in co-ordinate and momentum space, lead to a definite set of consistent formulae for divergent Fourier integrals regularized by two scale parameters, $\mu$ and $\nu$, which remove ultraviolet and infrared singularities, respectively.

The principle of invariance of the regularization allows us also to derive definitions for the tadpole-type integrals (without external momenta or co-ordinates), which intermix infrared and ultraviolet divergencies, by splitting the integrals up into a sum of separate infrared, ultraviolet, and regular items. However, the values for tadpole graphs of a complicated structure in higher orders of perturbation theory prove to be ambiguous, depending on the order of evaluation of their subgraphs. This ambiguity cannot be fully eliminated even if we try to somehow relate $\nu$ with $\mu$ (which makes low-order tadpole diagrams to be zero, but nevertheless, does not lead to a unique determination of higher-order graphs; moreover, any information about ultraviolet logarithms is completely lost then).

In two-dimensional $\sigma$ models, where intrinsic infrared divergencies at zero masses are present, the results of the extended differential renormalization disagree with the finite minimally renormalized results of dimensional regularization. The difference goes beyond the range allowed by renormalization-scheme arbitrariness in perturbation theory. For the $O(n)$ sigma model with a single coupling constant, the two-loop coefficient of the $\beta$ function turns out to be zero when we use the infrared extension of differen- 
tial renormalization. This contradicts the old well-established result which should be scheme-independent.

As concerns the use of the infrared $\tilde{R}$ operation in dimensional regularization, we have verified by direct two-loop calculations that in renormalizable theories $(\overrightarrow{\mathbf{n}}$-field in $D=2-2 \varepsilon$ and $\phi^{4}$ in $D=4-2 \varepsilon$ ) it successfully recovers ultraviolet logarithms in the finite parts of the diagrams with exceptional momenta, so that the $\beta$ function, calculated through the finite invariant charge, proves to be independent of any logarithms (of momenta and renormalization scales). It coincides with the result derived in the conventional way from the coefficients of the first singularity in $\varepsilon$, which does not depend on the choice of the momenta for logarithmically divergent diagrams.

The two-loop results of the infrared extension of differential renormalization in the $\phi_{(D=4)}^{4}$ theory agree with dimensional renormalization up to finite counterterms. However, higher-order logarithmically divergent artificial tadpole diagrams (resulting from infrared rearrangement) suffer from an irremovable ambiguity. Thus, calculations with exceptional momenta cannot be performed consistently above two loops.

Our final conclusion is that the program of constructing an invariant generalization of differential regularization and renormalization, to deal with infrared divergencies, has failed.

\section{References}

[1] D.Z.Freedman, K.Johnson and J.I.Latorre, Nucl.Phys. B371 (1992) 353

[2] K.G.Chetyrkin and F.V.Tkachov, Phys.Lett. B114 (1982) 133;

V.A.Smirnov and K.G.Chetyrkin, Teor.Mat.Fiz. 63 (1985) 462;

V.A.Smirnov, Renormalization and Asymptotic Expansions (Birkhäuser Verlag AG, Basel-Boston-Berlin, 1991)

[3] J.C.Collins, Nucl.Phys. B80 (1974) 341;

B.L.Voronov and I.V.Tyutin, Yad.Fiz. 23 (1976) 664

[4] G.'t Hooft, Nucl.Phys. B61 (1973) 455

[5] E.R.Speer, J.Math.Phys. 15 (1974) 1

[6] A.A.Vladimirov, Teor.Mat.Fiz. 43 (1980) 210

[7] K.G.Chetyrkin, S.G.Gorishny, S.A.Larin and F.V.Tkachov, Phys.Lett. B132 (1983) 351;

D.I.Kazakov, Phys.Lett. B133 (1983) 406;

H.Kleinert, J.Neu, V.Schulte-Frohlinde, K.Chetyrkin and S.Larin, Phys.Lett. B272 (1991) 39

[8] M.T.Grisaru, D.I.Kazakov and D.Zanon, Nucl.Phys. B287 (1987) 189

[9] A.A.Vladimirov, Teor.Mat.Fiz. 35 (1978) 392 
[10] P.Breitenlohner and D.Maison, Commun.Math.Phys. 52 (1977) 11

[11] L.Alvarez-Gaumé, D.Z.Freedman and S.Mukhi, Ann.Phys. 134 (1981) 85;

D.H.Friedan, Ann.Phys. 163 (1985) 318;

V.V.Belokurov and D.I.Kazakov, Fiz.Elem.Chast.Atomn.Yadra [Particles \& Nuclei] 23 (1992) 1322

[12] M.T.Grisaru, A.E.M.van de Ven and D.Zanon, Nucl.Phys. B277 (1986) 388; 409

[13] K.G.Chetyrkin, A.L.Kataev and F.V.Tkachov, Nucl.Phys. B174 (1980) 345;

K.G.Chetyrkin and F.V.Tkachov, Nucl.Phys. B192 (1981) 159

[14] D.I.Kazakov, Teor.Mat.Fiz. 75 (1988) 157

[15] E.Brézin and J.Zinn-Justin, Phys.Rev. B14 (1976) 3110;

J.Zinn-Justin, Quantum Field Theory and Critical Phenomena (Clarendon Press, Oxford, 1989) p.655

[16] G.M.Avdeeva, A.A.Belavin and A.P.Protogenov, Yad.Fiz. 18 (1973) 1309;

A.A.Vladimirov and D.V.Shirkov, Usp.Fiz.Nauk 129 (1979) 407 